\title{
Sujeitos no Exílio: implicações éticas e teológicas sobre a identidade dos refugiados em uma perspectiva pós-colonial.
}

\author{
Sujetos en exilio: implicancias éticas y teológicas sobre la \\ identidad de los refugiados desde una perspectiva poscolonial. \\ Subjects in Exile: ethical and theological implications on the \\ identity of refugees in a post-colonial perspective.
}

\section{Resumo}

O artigo é fruto de uma palestra dada na Alemanha sobre o tema dos refugiados e das migrações. Propõe diálogo, intercâmbio e aprofundamento de elementos analíticos neste tema a partir de diversas experiências que atravessam muitos contextos sóciopolíticos e culturais. Possui três partes. Na primeira, como marco geral, introduz os estudos pós-coloniais e outras subdisciplinas que se desprendem deles. Na segunda, indaga a respeito do espaço ontológico-político - a construção de identidades e o lugar da noção de sujeito - desde as dinâmicas de Estado-nação nos processos de globalização contemporâneos, utilizando pontos de vista latino-americanos. Por último, conclui com algumas reflexões sobre a necessidade de realizar práticas de re-territorialização da tradução cultural a partir de uma perspectiva ética, política e teológica.

Palavras-chave: Pós colonialismo; refugiados; migrações; sujeitos; identidades.

Licenciado em Teologia pelo Instituto Superior Evangélico de Estudos Teológicos - ISEDET (Argentina). Mestre em Antropologia Social e Doutor em Ciências Sociais pela FLACSO Argentina. Diretor geral do Grupo de Estudos Multidisciplinares sobre Religião e Incidência Pública (GEMRIP www.gemrip.org) e membro da direção da Postcolonial Network e da Fraternidade Teológica Latino-americana. (nicolaspanotto@gmail.com). 


\title{
Resumen
}

El artículo es el resultado de una conferencia dada en Alemania sobre el tema de los refugiados y la migración. Propone el diálogo, el intercambio y la profundización de elementos analíticos en este tema desde diferentes experiencias que cruzan muchos contextos sociopolíticos y culturales. Tie- ne tres partes. En el primero, como marco general, introduce los estudios poscoloniales y otras subdisciplinas que se derivan de ellos. En el segundo, pregunta sobre el espacio ontológico-político - la construcción de identida- des y el lugar de la noción de sujeto - desde la dinámica del estado-nación

en los procesos contemporáneos de globalización, utilizando puntos de vista latinoamericanos. Finalmente, concluye con algunas reflexiones sobre la necesidad de llevar a cabo prácticas de redistribución territorial de la traducción cultural desde una perspectiva ética, política y teológica.

Palabras-clave: Post colonialismo; refugiados; migraciones; sujetos; iden- tidades.

\begin{abstract}
The article is the result of a lecture given in Germany on the topic of refugees and migration. It proposes dialogue, exchange and deepening of analytical elements in this theme, based on diverse experiences that cross many so- cio-political and cultural contexts. It has three parts. In the first, as a general framework, it introduces post-colonial studies and other sub-disciplines that derive from them. In the second, he asks about the ontological-political space the construction of identities and the place of the notion of subject - from the dynamics of the nation-state in contemporary globalization processes, using Latin American points of view. Finally, it concludes with some reflections on the need to carry out practices of re-territorialization of cultural translation from an ethical, political and theological perspective.
\end{abstract}

Keywords: Post colonialism; refugees; migrations; subjects; identities.

\section{Introducción}

Me siento en un lugar complejo, siendo argentino -oriundo de la provincia de Santa Fe, o sea, la capital de una provincia a casi 600 kilómetros de Buenos Aires, donde -como dice el dicho- "Dios atiende el teléfono" sobre todo lo que acontece en el país-, latinoamericano, hablando a un público mayoritariamente europeo, sobre un tema tan coyuntural y sensible para este contexto como es el de la situación de los refugiados. Pero lo que puede parecer algo dispar desde lo formal, en realidad representa una situación mucho más cotidiana, común y real dentro de nuestros con- 
textos de que lo a veces queremos reconocer.

Afirmo esto último por dos aspectos. Primero, por el evidente factor de que las dinámicas migratorias y los conflictos sociopolíticos y culturales que ello despierta en diversos contextos nacionales, es una situación muy real en toda América Latina. El caso más conocido es el de la frontera México-EE. UU., que en realidad no se relaciona sólo con mexicanos/as sino con grupos que provienen de varios países de América Central y que experimentan tortuosas y fatales odiseas para llegar a la frontera en busca de un mejor destino. Pero también existen muchos otros casos no tan mediáticos pero que determinan muchas dinámicas socio-políticas en la región, como son las migraciones de países andinos o Paraguay a Argentina y Uruguay, los refugiados cubanos que llegan a EE. UU., las migraciones de 
grandes grupos colombianos a distintos países del continente y a Canadá tras los conflictos con los paramilitares o la guerrilla, los conflictos raciales que se viven en Brasil que producen continuos movimientos internas, los procesos migratorios que emergen a raíz de los conflictos entre maras y narcotraficantes en El Salvador y Honduras, los inmigrantes haitianos que llegan a diversos países pero principalmente a República Dominicana, Panamá y Nicaragua, y por último la situación creciente de la llegada de africanos al Cono Sur. Esto sin contar los movimientos constantes de latinoamericanos en todo el mundo -especialmente a Europa y Estados Unidos-, como también los procesos de circulación constante entre centros urbanos y periferia rural, estructura que refleja un claro enclave colonial que aún persiste en nuestros países, donde las ciudades capitales son el epicentro del poder político, económico, académico y social.

Pero en segundo lugar -y como resultado de lo recién descrito-, todas estas dinámicas -tanto en América Latina como en Europa- deben inscribirse dentro de marcos de análisis más amplios, que provienen no sólo de situaciones similares compartidas sino también de la expansión de diversos imaginarios con respecto a la migración y los refugiados que se comparten a nivel global, y la necesidad de ubicar estos fenómenos desde estudios con mayor alcance histórico, contextual y geopolítico. En este sentido, este encuentro puede resultar en un interesante ejercicio de diálogo, intercambio y profundización de elementos analíticos que atraviesan todos los contextos, y que pueden ser enriquecidos en su capacidad de lectura a partir de todas las experiencias aquí presentes.

Esta ponencia se dividirá en tres partes. Primero, introduciremos los estudios poscoloniales y otras subdisciplinas que se desprenden de ellos, como marco general de la presentación. Segundo, indagaremos en torno al espacio ontológico-político -o sea, la construcción de identidades y el lugar de la noción de sujeto- desde las dinámicas de Estado-nación dentro de los procesos de globalización contemporáneos, utilizando algunos trabajos de corte latinoamericano. Por último, concluiremos con algunas reflexiones sobre la necesidad de realizar prácticas de re-territorialización de la traducción cultural desde una perspectiva ética, política y teológica.

\section{Dinámicas y sentidos geopolíticos desde una perspectiva poscolonial}

Queremos enmarcar nuestro abordaje desde lo que entendemos como teoría o abordaje poscolonial. ¿Qué es la teoría poscolonial? Dos elementos para resumirlo. Por un lado, este término remite a una nominación sociohistórica. El poscolonialismo plantea que a pesar de los procesos de independencia nacionales en las colonias como también las transformaciones en las configuraciones geopolíticas en las últimas décadas, aún persisten dinámicas coloniales, no sólo en lo que refiere al campo de la econo- 
mía mundial sino también sociopolíticas (la presencia de Estados-nación), socioculturales (como las formas de definir ciudadanía) y el lugar de lo religioso. Una de las nociones centrales en esta dirección es la de imperialismo, pero comprendida no como un modo de poder centralizado en un país específico o desde una división bipolar de la realidad global sino como un conjunto de fluidos que circulan y se reproducen a través de las dinámicas sociales a nivel transnacional (Hardt y Negri, 2000)

Uno de los temas centrales para esta corriente es la dinámica del othering, o sea, la manera en que Occidente construye sus "otros" para cual teoría del espejo lacaniana- conformar y legitimar la clausura y sutura de su propia (falsa) homogeneidad identitaria. La obra más conocida al respecto es Orientalismo del filósofo palestino Edward Said (2003), quien realiza una profunda crítica a los estudios orientales europeos, y ofrece una relectura filosófica de corte sociopolítico y literario donde demuestra cómo la academia y política occidentales sintomatizan el llamado "Oriente" a partir de diversos estigmas y preconceptos identitarios, que en realidad sirven como una forma de circunscribir una frontera que enuncia su propia identidad. Como demuestra Anibal Quijano (2000), la división Oriente-Occidente trae aparejado consigo otros binomios como son primitivo-civilizado, mágico/mítico-científico, racional-irracional, tradicional-moderno. O sea, Europeo-No europeo.

En segundo lugar, el poscolonialismo también evoca a un marco epistemológico y metodológico que se alinea con diversos abordajes posmodernos y posestructuralistas, que priorizan categorías analíticas como el estudio de los procesos de identificación, el lugar del análisis de los discursos en la construcción de procesos socioculturales, y la deconstrucción de imaginarios y prácticas sociopolíticas. En este sentido, intenta superar ciertos reduccionismos estructuralistas y lecturas bipolares que simplifican las complejas dinámicas de poder, y que por ello no facilitan una lectura lo suficientemente profundizada de la acción de los sujetos y la heterogeneidad de sectores y grupos, que presionan, resisten y negocian con las fronteras que enmarcan modos de institucionalidad másamplios.

La teoría poscolonial tiene muchos derivados disciplinares, aunque en este caso nos importa mencionar tres. Primero, el giro decolonial cuyo origen se encuentra en el Grupo de Estudios Modernidad/Colonialidad (Escobar, 2003), compuesto por académicos como Aníbal Quijano, Walter Mignolo, Enrique Dussel, Arturo Escobar, Catherine Walsh, entre otros/ as. Este grupo comenzó con un abordaje centrado en el contexto de América Latina, aunque fue ampliándose poco a poco como una propuesta teórica con cierta autonomía. Intenta diferenciarse de los abordajes clásicos poscoloniales, a partir de su cuestionamiento al trasfondo occidental que poseen los estudios poscoloniales. Por otro lado, también realizan un mayor trabajo de análisis socio-antropológico a través de teorías políticas 
y económicas, y no tanto dentro de la crítica literaria, como suelen ser los estudios poscoloniales.

Otras dos corrientes importantes vinculadas a los estudios poscoloniales son los border studies, que amplían el abordaje sobre las implicancias socio-cutlurales de las fronteras políticas, culturales de identitarias, las cuales no sólo representan una demarcación de naciones sino "una suma de procesos sociales, políticos y culturales" (Ladino y Gil, 19) Más adelante ampliaremos este abordaje. Por último, también encontramos los diasporic studies, los cuales tienen un trasfondo dentro de los estudios religiosos, especialmente dentro de la historiografía judía. Estos trabajos han dado gran énfasis al estudio literario y a la crítica hermenéutica -que desde una perspectiva posmoderna no implica sólo una instancia de análisis de discurso sino, a partir de él, de crítica sociopolítica-, específicamente desde la experiencia diaspórica como instancia de hibridez cultural.

Para resumir, el abordaje poscolonial nos sirve para lo siguiente:

1. Hacer un abordaje más complejo de las dinámicas socioculturales dentro de la globalización, superando las lecturas estructuralistas y economicistas, a través del análisis de los procesos heterogéneos y ambivalentes que se dan entre procesos culturales, discursivos, comunicacionales, relacionales, etc.

2. Ubicar las prácticas miméticas de los sujetos (Bahbah, 1994), o sea, verlos no sólo como víctimas pasivas de un sistema omnipresente sino como sujetos que constantemente negocian y resignifican marcos de poder a partir de su especificidad sociocultural.

3. Analizar lo cultural como un encuentro intercultural y no como un "choque de civilizaciones".

\section{Sujetos en exilio: ontología política en la encrucijada entre Estados-nación y globalización}

La globalización plantea una situación ambivalente y paradójica. Por un lado, busca la apertura y acceso total a través de los medios de comunicación, y por otro facilita procesos cada vez más severos de restricción y control fronterizo. Los discursos de integración que se proponen como alternativas utópicas tanto para enfrentar el problema de la desintegración-Mercosur, ALCA, UE- como para luchar contra las lógicas imperiales -la "Patria Grande"-, también son marcos de sentido funcionales que carecen de reconocimiento y facilitan la exclusión de la diversidad. Como dice el antropólogo Cardoso de Oliveira, estas cosmovisiones olvidan la realidad de la fricción interétnica constitutiva de cualquiercultura.

Profundizando más en esta dirección, por un lado emerge un gran problema a la hora de vincular el Estado-nación con lo identitario. Uno de los puntos de partida centrales de la crítica decolonial es la denuncia sobre la relación que existe entre colonialismo y modernidad. Son dos 
caras de la misma moneda. Esto indica que más allá de la anulación de ciertas dinámicas de colonialismo directo, persisten ciertas prácticas e institucionalidades que aún mantienen lógicas coloniales, entendidas como la promoción de visiones occidentales tanto a nivel identitario como de procesos sociales.

En esta dirección, es interesante el análisis que hace Hanna Arendt (1998): el juicio ético frente a la falta de Estado no deviene de la falta de civilización sino que representa un síntoma de la propia modernidad. En otras palabras, para la modernidad no hay forma de "ser" sin Estado; o sea, apátrida. De la misma manera, los apátridas no se encuentran en la periferia o los márgenes de la sociedad sino que dentro de la condición global. Como dice Edward Said (Cultura e imperialismo, 495ss.), la resistencia a lo inmigratorio y a los refugiados deviene no de una razón pragmática de contención sino de la amenaza que se percibe hacia la estabilidad del Estado-nación en tanto marco identitario.

En resumen, podemos decir que los conflictos que producen la presencia de refugiados, inmigrantes y exiliados provienen no de una limitación estructural de un país, sino que son respuestas frente a un síntoma de doble origen: los sentimientos de amenaza frente a la seguridad que produce la homogeneidad y pertenencia a un sentido de nacionalidad, y al rechazo frente a las consecuencias contradictorias que producen las acciones de los estados-nación a nivel geopolítico, como son la venta armamentística, el apoyo de conflictos regionales (Medio Oriente, África, Centro América y países andinos), el boicoteo constante a economías locales que producen pobreza y falta de desarrollo interno, etc.

Como indica el subtítulo, el problema en este contexto se vincula con las construcciones ontológicas, o sea, con los sentidos de identificación que median en los procesos sociales, que en este caso responden a cierto enclave colonial. En otros términos, los procesos coloniales presentes en las dinámicas son legitimados por y a su vez promueven modos de comprensión de y acercamiento a la otredad. Por esta razón, el ejercicio de deconstrucción de sentidos es elemental a la hora de plantearnos cómo enfrentar los nuevos escenarios que nos presentan los procesos actuales de migración. 1Qué significa ser ciudadano? lQué lugar tiene el sentido de nacionalidad en este contexto? ¿Qué estatus sociopolítico poseen los refugiados? ¿Desde dónde determinarlo? ¿Dónde reside el derecho y la legalidad? ¿Cómo devienen las dinámicas de resistencia por parte de las sociedades frente a este contexto?

\section{Espacialidad fronteriza y dislocación sociocultural}

Uno de los desafíos en este contexto es superar lo que se denomina nacionalismo metodológico, el cual define la identidad de un grupo o territorio a partir de la inscripción de ciertos imaginarios patrióticos, que ob- 
viamente responden a la inscripción en un estado-nación. Por esta razón, los refugiados presentan un desafío a la homogeneidad identitaria, que no sólo respalda un sentido de pertenencia territorial sino también un conjunto de dinámicas de poder sociopolíticas y económicas.

Los border studies han contribuido a una crítica de estas nociones a través de la deconstrucción del sentido de frontera. Ella no es una línea de delimitación entre territorios, sino que representa espacios de "intercambio, contacto, mezcla o encuentro" (Rodas, 2011) Las fronteras poseen una significación política, estratégica y simbólica. De aquí la duplicidad del concepto de frontera: fue y es un objeto/concepto y un concepto/metáfora. En el primer caso refiere a las fronteras físicas, en el segundo a las culturales (Grimson, 2000) Esta dualidad no refiere sólo a la división entre dos objetos/naciones/Estados sino a las fronteras internas de los propios marcos identitarios.

De aquí que algunos prefieren hablar más bien de zonas fronterizas o espacios liminales: lugares donde las identidades y los sujetos circulan a través de las negociaciones, resistencias y resignificaciones de los sentidos que despiertan y movilizan los delineamientos culturales. Más aún, esta dinámica fronteriza (Mignolo) no sólo refiere a la enmarcación de lo territorial sino a la comprensión de la misma identidad, la cual no se comprende como un todo suturado por fronteras que circunscriben un sentido homogéneo, sino más bien como un conjunto de procesos polivalentes en constante tensión. Esta misma paradoja y ambivalencia proyectan sentidos sedimentados pero inscriptos en una espacialidad plural y heterogéneas que hacen a un movimiento constante.

En conclusión, lcómo se definen los "objetos" que dividen una frontera? ¿Hasta qué punto están delimitados? Si las fronteras son espacialidades de circulación e intercambio simbólico, ¿dónde se deposita "lo cultural" en dicho encuentro? Como propone la teoría poscolonial, inclusive la distinción entre "local" y "global" no logra representar la complejidad de estos procesos; más aún, en dicha diferenciación se sigue manteniendo la clausura de dinámicas que se muestran homogéneas (o sea, lo global y lo local como entidades cerradas e identificables), y con ello respalda instancias opresivas de poder.

Por esta razón, se habla de la dialéctica contingente como manera de leer la constitución de la identidad sociocultural y sus procesos de interacción. Este concepto supera la comprensión de las diferencias como circuitos autónomos y aislados, como también el hablar de un proceso global ad extra, como una especie de entidad supra histórica a las dinámicas socioculturales específicas, corporales y relacionales. Más bien, antes que hablar de "cultura" como entidad suturada, deberíamos referirnos a lo cultural como una dinámica que más allá de responder a ciertas delimitaciones (lingüísticas, históricas, territoriales, contextuales), son renego- 
ciadas constantemente a través del encuentro con el otro. Como concluye Grimson (2011:146):

No hay culturas en diáspora, ya que eso equivaldría a afirmar que hay un grupo culturalmente homogéneo que genera enclaves clonados de diferencia en distintas zonas del planeta. La diáspora es una configuración cultural transnacional, un espacio de heterogeneidad articulado, una de cuyas condiciones necesarias es la identificación compartida.

\section{La riqueza del encuentro intercultural}

Las teorías poscoloniales plantean que la diferenciación cultural se da también en los encuentros coloniales, o sea, entre los Estados-nación o empresas imperiales y grupos subalternos (apátridas, refugiados, inmigrantes). En dicha diferenciación, no sólo se juega una dinámica de opresión sino también de alteridad, donde las identidades subalternas no sólo son subsumidas bajo la mano del opresor, sino que producen un espacio de renegociación, resistencia y resignificación de las fronteras que le son impuestas (Bahbah, 1994) Aquí dos elementos: ni las fronteras son fijas, ni los sujetos pasivos. Toda diferenciación que se gesta, más allá de los niveles de coacción, siempre producirá un proceso dialéctico intercultural que facilitará la deconstrucción de sentidos como de resistencias y enriquecimientos mutuos.

Aquí uno de los aspectos que las teorías de/poscoloniales y los border studies plantean: todo encuentro debe ser valorado desde la riqueza cultural que propone y no como una amenaza frente a la identidad propia (Castillo, El rostro humano de la migración) Como dice Walter Mignolo, a diáspora crea un locus de enunciación crítica. Por su parte, Luis Rivera-Pagan (2002:5) afirma que:

the displacement of migration creates a new space of liberation from the atavistic constraints and bondages of the native cultural community and open new vistas, perspectives, and horizons.

Enfatizar este elemento no pretende obviar los conflictos inherentes a estos procesos ni las condiciones de opresión que se juegan. Más bien, evidencia un proceso de mímesis donde los contextos de opresión son resignificados en el encuentro liminal. Reconocer este elemento permite promover y construir otros sentidos que se juegan en el encuentro, lo cual permite deconstruir y cuestionar los estigmas con los que se cargan a refugiados e inmigrantes. Por otro lado, también presiona la homogeneidad de las fronteras nacionales, lo que permite imaginas nuevos enclaves identitarios, que se reflejen a su vez en nuevas políticas públicas y prácticas ciudadanas. Los estudios decoloniales lo denominan de-linking: los desprendimientos que se dan desde los marcos cerrados por la presión de lo subalterno, lo diferente, lo excluido interno. Como concluye Said (Exilio,502): 
La gran tarea, entonces, es combinar los nuevos dislocamientos y configuraciones económicas y sociopolíticas de nuestra época y la asombrosa realidad de la interdependencia humana en escala mundial.

\section{En busca de reterritorializaciones en la traducción cultural: perspectivas éticas, políticas y teológicas}

Resumiendo lo elaborado hasta aquí, podemos afirmar lo siguiente:

1. El fenómeno de los refugiados y la inmigración representa un síntoma de las propias políticas coloniales de Occidente, cuyos conflictos inherentes despiertan una actitud de clausura y condena sociocultural frente a este fenómeno, lo que expone el lado más oscuro de un posicionamiento que se cree neutral o fortuito; o sea, evadiendo las consecuencias de su colonialismo inherente y escondido.

2. Los nacionalismos y las definiciones jurídicas de lo ciudadano en el marco del Estado-nación representan resabios de las lógicas coloniales occidentales, los cuales clausuran formas diversas de definición de lo identitario, provocan resistencias y rechazos, como también el reconocimiento de la heterogeneidad que compone cualquier sedimentación sociocultural.

3. Un principio elemental para la construcción de instancias alternativas de resignificación es la deconstrucción de imaginarios estigmatizantes a partir de una noción de lo identitario que no se defina a partir del Estado-nación sino a través del encuentro intercultural.

Frente a esto, podemos pensar en algunos elementos a nivel ético, sociopolítico y teológico-religioso que nos permitan profundizar en una repuesta. Para ello, me gustaría partir de la idea que propone Homi Bahbah (2010:79-87) sobre el sentido del tercer espacio. Este concepto deviene de diversas experiencias con los modos de interpretar sentidos y palabras durante instancias de traducción, donde aparecen gestos, palabras y objetos que cuestionan los sentidos dados y permiten otros nuevos. Es un lugar dialógico, un espacio intercultural de enunciación, donde los sentidos son relativizados, a través de un encuentro cara a cara.

La búsqueda de este tercer espacio requiere de una búsqueda por empoderar nuevas narraciones. Narraciones que parten del diálogo, o sea, de un acercamiento concreto, real y corporal con el otro. Un cara a cara que escucha, que permite enunciar y que nos resignifica. Un apalabramiento que -en términos de Deleuze- produce reterritorializaciones, o sea, nuevos locus de sentido, elemento que en este caso podríamos hablar como de nuevos territorios de ciudadanía, con otras vas fronteras surgidas del intercambio. En palabras de Bahbah (2013:56-57): 
Las lecciones de la ambivalencia no se agotan en el acto de soportar. La experiencia de lo ambivalente resulta también un acicate para el discurso, la necesidad de la palabra, una vía para trabajar lo contradictorio y lo que no tiene solución, con el propósito de lograr el derecho a narrar. Incluso las formas más extremas de ambivalencia cultural... constituyen momentos que coaccionan el acto de soportar hasta convertirlo en reclamo por la agencia de discurso e interlocución.

Volviendo al título de esta ponencia, desde lo expuesto el exilio no lo veríamos como un accidente fortuito sino como un locus constitutivo de toda identidad. En ese exilio se dan situaciones de opresión, injusticia, desolación. Pero el problema no se deposita en el exilio en sí, desde un juicio moral que parte desde el sentimiento de amenaza a una concepción colonial de apátrida. El problema, por el contrario, se ubica en la rigidez de posicionamientos que impiden el proceso, que facilitan la expulsión como síntoma de su opresión y que anulan la noción de proceso, de caminata, de movimiento, por temor de perder la seguridad de la falsa homogeneidad identitaria.

Por ello, podemos partir de la búsqueda de un tercer espacio en tres campos particulares:

1. En la construcción de espacios de diálogo intercultural, donde las barreras identitarias sean levantadas en pro de la liberación de procesos de identificación enriquecidos por el intercambio. Esto significa cuestionar las visiones nacionalistas y puristas, que se traslucen no sólo en discursos condenatorios explícitos sino también en prácticas y gestos cotidianos hacia "los otros". ¿Cómo podemos pensar las comunidades de fe como espacios de diálogo y encuentro?

2. El tema religioso es uno de los más sensibles en estos procesos. Lo vemos con el islam en Europa, y las religiosidades indígenas o populares en América Latina. Las nociones de laicidad/laicismo infieren tipos de acercamientos al inmigrante. Una clausura en este campo también implica una clausura en el acercamiento hacia el otro cultural, más aún desde la comprensión esencialista sobre lo religioso que persiste en occidente. ¿Cuáles son las nociones de (pós)secularización que median en los diálogos interculturales?

3. Desde la perspectiva de la fe, requerimos de nuevos acercamientos teológicos en diversas direcciones:

a. Una profundización en las implicancias sociopolíticas y culturales de una teología sensible al pluralismo religioso. Cuando hablamos de teología, no sólo nos referimos a deconstrucciones dogmáticas sino más aún en la comprensión y definición 
de lo divino per se. Como demuestran los estudios poscoloniales, las misiones cristianas y el sentido de destino manifiesto de las naciones imperiales, han promovido una visión colonizadora de lo divino a partir de una noción esencialista de lo otológico. La clausura identitaria de occidente decayó sobre Dios mismo. Si lo cultural es una mediación central para definir lo divino, lqué significa hablar de Dios en la liminalidad, en el in-between (Bahbah) de los procesos culturales? lPodríamos pensar en un Dios que se manifiesta en la fisura que imprime la diferencia?

b. El tema del exilio es un tema bíblico elemental. Los estudios de la diáspora dentro de la exégesis bíblica permiten visualizar cómo los procesos de movimientos de personas y comunidades tienen fuerte impacto en la hermenéutica bíblica. En este sentido, el reconocimiento de un proceso de traducción cultural a partir del texto bíblico también impulsaría la "invitación" a un espacio de construcción de nuevas "zonas fronterizas" donde los sentidos emergen de lo fronterizo (Mignolo).

Para concluir, reflexionemos sobre las siguientes palabras de Gloria Anzaldúa, académica chicana:

A borderland is a vague and undetermined place created by the emotional residue of an unnatural boundary. It is an a constant state of transition. The prohibited and forbidden are its inhabitants. Los atravesados live there: the squint-eye, the perverse, the queer, the throublesome, the mongrel, the mulato, the half-breed, the half dead; in short, those who cross over, pass over, or go through the confines of the normal (Anzaldúa 2007:25)

En línea con lo que hemos trabajado en esta exposición -y tal como mencionamos al inicio-, Anzaldúa nos muestra que los procesos de conflicto en torno a la delimitación legal y política de las fronteras, el rol del Estado-nación, los prejuicios con respecto a los refugiados, etc., responden a comprensiones más amplias a nivel sociocultural, desde factores mucho más allá -en lo histórico, lo social, lo religioso, lo político- de las contingencias geopolíticas contemporáneas. En otras palabras, las dinámicas que vivimos hoy deben entenderse como síntoma de prácticas y sentidos legendarios de la impronta colonial de occidente, como también de prejuicios, estigmas y preconceptos sobre "lo raro", lo diferente, lo extraño, que siempre se presenta como una amenaza a la sutura cultural de nuestras sociedades. Por ello, un proceso de reconstrucción de sentidos y de nuevas prácticas de relacionamiento y traducción intercultural debe ser uno de los primeros pasos a dar, para que las políticas públicas e internacionales no caigan en saco roto.

Universidad Augustana, Alemania - 13 de mayo 2016 


\section{Bibliografía}

Anbzaldúa, Gloria (2007) Borderlands/La Frontera. The New Mestiza. San

Francisco: Aunt Like Books

Arendt, Hannah (2013) La condición humana. Buenos Aires: Paidós

Bhabha, Homi (1994) El lugar de la cultura. Manantial: Buenos Aires

-- (2013) Nuevas minorías, nuevos derechos. Siglo XXI: Buenos Aires

Escobar, Arturo (2013) "Mundos y Conocimientos de otro modo" En: Ta-

bula Rasa. Bogotá - Colombia, No. 1: 51-86, enero-diciembre de 2003

Grimson, Alejandro (2000) Fronteras, naciones e identidades. La periferia como centro. La Crujía: Buenos Aires

-- (2011) Los límites de la cultura. Siglo XXI: Buenos Aires

Mignolo, Walter (2013) Historias locales/diseños globales. Akal: Madrid

Quijano, A. (2000). "Colonialidad del poder y clasificación social". Journal of World-Systems Research, VI (2), 342-386.

Rivera-Pagán, Luis (2002) Essays from the Diaspora. Publicaciones El Faro: México

Said, Edwars (2005) Reflexiones sobre el exilio. Debolsillo: Buenos Aires

-- (2011) Cultura e imperialismo. CompanhiaDeBolso: San Pablo 